\title{
A quantitative property-property relationship for the internal diffusion coefficients of organic compounds in solid materials
}

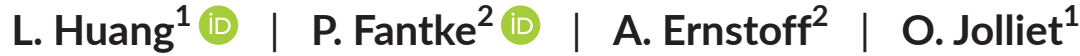

${ }^{1}$ Department of Environmental Health Sciences, School of Public Health, University of Michigan, Ann Abor, MI, USA

${ }^{2}$ Division for Quantitative Sustainability Assessment, Department of Management Engineering, Technical University of Denmark, Lyngby, Denmark

\section{Correspondence}

Lei Huang, Department of Environmental Health Sciences, School of Public Health, University of Michigan, Ann Abor, MI, USA. Email: huanglei@umich.edu

Funding information

US EPA, Grant/Award Number: EP16-C-000070; Long Range Research Initiative of the American Chemistry Council; Marie Curie project Quan-Tox; European Commission; Seventh Framework Programme, Grant/Award Number: 631910.

\begin{abstract}
Indoor releases of organic chemicals encapsulated in solid materials are major contributors to human exposures and are directly related to the internal diffusion coefficient in solid materials. Existing correlations to estimate the diffusion coefficient are only valid for a limited number of chemical-material combinations. This paper develops and evaluates a quantitative property-property relationship (QPPR) to predict diffusion coefficients for a wide range of organic chemicals and materials. We first compiled a training dataset of 1103 measured diffusion coefficients for 158 chemicals in 32 consolidated material types. Following a detailed analysis of the temperature influence, we developed a multiple linear regression model to predict diffusion coefficients as a function of chemical molecular weight (MW), temperature, and material type (adjusted $R^{2}$ of .93 ). The internal validations showed the model to be robust, stable and not a result of chance correlation. The external validation against two separate prediction datasets demonstrated the model has good predicting ability within its applicability domain $\left(R_{\text {ext }}^{2}>\right.$. $)$, namely $\mathrm{MW}$ between 30 and $1178 \mathrm{~g} / \mathrm{mol}$ and temperature between 4 and $180^{\circ} \mathrm{C}$. By covering a much wider range of organic chemicals and materials, this QPPR facilitates high-throughput estimates of human exposures for chemicals encapsulated in solid materials.
\end{abstract}

\section{KEYWORDS}

consumer products, correlation, diffusion, indoor release, organic chemicals, solid materials

\section{1 | INTRODUCTION}

Chemicals encapsulated in solid materials have been identified as a major source of passive emissions to indoor air $^{1-3}$ and of transfers into food $^{4}$ and onto skin. ${ }^{5}$ Typical examples include chemicals used as flame retardants in furniture and plasticizers in food contact materials (FCMs). To estimate the release of these chemicals from solid materials, and eventually consumer exposures, the diffusion coefficient, $D\left(\mathrm{~m}^{2} / \mathrm{s}\right)$, for chemicals encapsulated in solid materials, is essential information. $D$ describes the transport of a molecule through a material, which is specific for a chemical-material combination and is also influenced by ambient temperature. Experimental techniques such as chamber tests for building materials, ${ }^{6,7}$ and sorption/desorption experiments for polymer materials ${ }^{8-10}$ have enabled measurement of a limited number of chemical diffusion coefficients for building materials such as vinyl flooring, gypsum board, particle board, plywood, carpet, and cement, ${ }^{11-14}$ as well as polymer materials including polyethylene (PE), polystyrene (PS), polypropylene (PP), and polyvinyl chloride (PVC). ${ }^{4,15,16}$ However, given the limited number of chemical-material combinations with measured $D s$, and the costly and time-consuming nature of experiments, quantitative relationships are needed to complement existing measurements by predicting the diffusion coefficients from known physiochemical properties for chemicals without experimental data. This is especially important for high-throughput approaches where a large number of chemical-material combinations need to be evaluated and for which it is unrealistic to perform experiments on all relevant combinations. 
Several correlation methods have been developed to estimate the diffusion coefficients from physicochemical properties of chem-

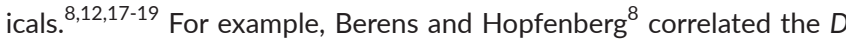
to the mean molecular diameter of the diffusing molecule, using data on more than 20 chemicals in three glassy materials including PVC, PS, and polymethyl methacrylate (PMMA). Zhao et al. ${ }^{19}$ found a correlation between $D$ and vapor pressure for water and eight aromatic hydrocarbons in polyurethane foam (PUF). Furthermore, both Bodalal et al. ${ }^{12}$ and Cox et al. ${ }^{18}$ estimated the $D$ as a function of molecular weight. The former study considered measured $D$ data on five aromatics and five aldehydes in several building materials, ${ }^{12}$ while the latter study considered data on four alkanes in vinyl flooring. ${ }^{18}$ For each of these aforementioned approaches, the main limitation is that the correlations are specific to certain chemical classes and materials, for example, aldehydes in plywood, which limits their application for other materials and chemical classes. Addressing this research gap to facilitate wider applicability, Guo ${ }^{17}$ developed a method which estimates the diffusion coefficient as a function of the chemical's molar volume for mixed chemical classes. However, this approach is limited to six building materials and is developed based on a small dataset of limited chemical classes ( $\leq 3$ chemical classes for five of the six building materials).

The aforementioned correlation methods consider experiments for building materials at room temperature, and therefore, temperature is not relevant and thus not considered in the correlation model. For other exposure scenarios, such as transfer of chemicals from FCMs into food, ambient temperature is highly relevant because FCMs can be heated, refrigerated, or frozen. Accordingly, Begley et al. ${ }^{4}$ presented a correlation method to estimate the diffusion coefficient in nine polymer materials as a function of molecular weight and temperature, which is not applicable beyond the considered polymers.

In all, the currently available correlation methods to estimate $D$ do not provide sufficient coverage of chemicals encapsulated in consumer products in different use scenarios (ie, ambient temperatures). Developing low-tier, high-throughput methods to estimate exposure to chemical in consumer products across a variety of chemicalmaterial combinations is a recent focus in various science-policy fields such as computational exposure science and life cycle assessment (LCA). ${ }^{20-25}$ Addressing the lack of methods to estimate $D$ for a variety of chemical-product scenarios, this study aims to develop a more comprehensive correlation method to estimate $D$ for wide range of organic compounds in multiple solid materials. More specifically, we aim to:

- carry out a comprehensive and extensive literature review to collect experimental diffusion coefficient data on a wide range of materials and chemicals.

- use multiple linear regression (MLR) techniques to establish the relationship between the diffusion coefficient and various predictor variables including physiochemical properties, material properties, and environmental characteristics.

- perform internal and external validations to characterize the validity and predictive power of the developed correlation.

\section{Practical Implications}

- The quantitative property-property relationship developed by this study provides a more comprehensive correlation method to estimate the diffusion coefficients, as it covers a wide range of organic chemicals and solid materials, and also considers the effect of temperature. This model provides the basis for facilitating high-throughput estimates of indoor human exposures for chemicals encapsulated in solid materials relevant for several science-policy fields, such as chemical alternatives assessment (CAA), risk assessment (RA), and life cycle assessment (LCA).

As the material type is a categorical property variable and is not related to the chemical's molecular structure, we call this correlation a quantitative property-property relationship (QPPR) instead of a quantitative structure-activity relationship (QSAR). This QPPR provides a more advanced correlation method to estimate the diffusion coefficients of organic compounds compared to previous studies, as it covers a wide range of solid materials and physiochemical properties, and also considers the effect of temperature. By providing reliable estimates of this key diffusion parameter for a large number of chemicals, this method will facilitate high-throughput assessments of chemical emissions and human exposures for chemicals encapsulated in solid materials relevant for chemical alternatives assessment (CAA), risk assessment, and LCA.

\section{2 | MATERIALS AND METHODS}

\section{1 | Dataset}

Experimental diffusion coefficient data were compiled from 68 references from the peer-reviewed scientific literature. The initial dataset contained a total of 1124 records covering 161 unique chemicals and 88 distinct solid materials (provided in Appendix S2). Experimental data expressed in $\mathrm{cm}^{2} / \mathrm{s}$ were converted to $\mathrm{m}^{2} / \mathrm{s}$. There are different types of diffusion coefficients reported in the literature, so harmonization of these data was performed to develop a consistent dataset. For diffusion coefficients measured in liquid sorption experiments, the "intrinsic" diffusion coefficients, corrected for the swelling of materials, were collected. ${ }^{10}$ Sorption of the liquid molecules inside the solid material may cause swelling of the material, which would lead to decreased observed diffusion coefficients and thus need to be corrected. ${ }^{10}$ For porous materials consisting of pore space and solid material, two types of models can be used to describe the chemical transport through these materials. The one-phase model considers the porous material as an assumed homogeneously mixed material, so an "apparent" diffusion coefficient is used to describe the chemical diffusion through such imaginary material. ${ }^{7}$ In contrast, the multiphase model considers the material as a mixture of pores and solid parts, and the chemical diffuses mainly through the pores if the pores are 
interconnected, or through the pores and solid parts alternately if the pores are isolated from each other. The gas-phase diffusion through the pores, which can be described by an "effective" diffusion coefficient, is assumed to be much faster than the diffusion through the solid parts. ${ }^{7}$ Haghighat et al., ${ }^{7}$ has demonstrated that the "apparent" diffusion coefficient is equivalent to the "effective" diffusion coefficient $\left(D_{\mathrm{e}}\right)$ divided by the material phase-gas phase partition coefficient $\left(K_{\text {ma }}\right)$. Thus, for porous materials the "apparent" diffusion coefficients reported in studies were collected. ${ }^{26}$ For studies where only the $D_{e}$ and $K_{\mathrm{ma}}$ were reported, ${ }^{27-29}$ they were converted to "apparent" diffusion coefficients using the aforementioned method. Data were excluded for studies where only the "effective" diffusion coefficients were reported.

From the initial dataset, 21 records were excluded from further analyses because they involve chemicals that are inorganic, chemicals for which no CAS number could be identified, or chemicals that are polymer chains with varying molecular weights. The final considered dataset thus includes 1103 records for 158 unique chemicals and 87 materials.

\subsection{Modeling methods}

\subsection{1 | Multiple linear regression}

An MLR analysis was performed to identify and quantify the effect of different parameters on the diffusion coefficient. The MLR model takes the following general form:

$$
\log _{10} D=\alpha+\beta_{1} \cdot X_{1}+\cdots+\beta_{n} \cdot X_{n}+b_{1} \cdot M_{1}+\cdots+b_{m} \cdot M_{m}
$$

where $\log _{10} D$ is the logarithm of the diffusion coefficient $\left(\mathrm{m}^{2} / \mathrm{s}\right), \alpha$ is the intercept; $X_{1}$ to $X_{n}$ are independent variables related to physiochemical properties, such as molecular weight, molar volume, and vapor pressure, and/or environmental characteristics like temperature; $\beta_{1}$ to $\beta_{n}$ are regression coefficients for the respective independent variables $X_{1}$ to $X_{n}$; and $M_{1}$ to $M_{m}$ are dummy variables for the solid materials, with one dummy variable per type of material. A dummy variable equals 1 for the material type it represents, and equals 0 for all other materials; for example, $M_{1}=1$ for material type $=1, M_{1}=0$ for material types 2 to $m$. $b_{1}$ to $b_{m}$ are regression coefficients for the respective dummy variables $M_{1}$ to $M_{m}$. The number of $m$ is equal to the number of material types considered -1 , as the material type with the highest number of measured $D$ data is used as the reference material type and does not require a dummy available in the MLR. Note that the MLR model gives one coefficient for each material type, while a material type can represent a single pure substance such as calcium silicate, a composite material such as vinyl flooring and gypsum board, or a group of similar materials such as wooden boards. Details of the material types will be discussed later. This regression equation also implies that the material coefficients $\left(b_{1}\right.$ to $\left.b_{m}\right)$ and the physiochemical property coefficients $\left(\beta_{1}\right.$ to $\left.\beta_{n}\right)$ are independent of each other, which if corroborated by internal and external validations (Section 2.3), allow for the maximum prediction coverage in terms of chemical-material combinations. All regression coefficients were estimated by the least squares (LS) method. All regression analyses were performed using IBM SPSS Statistics version 23 (IBM corporation, Armonk, NY, USA).

\subsection{2 | Grouping of materials and initial regressions}

To reduce the number of dummy variables, to avoid over-fitting of the MLR model, and to have a minimum of 10 records and three different chemicals per material type to ensure enough variability, the 87 original materials were grouped into 32 consolidated material types, based on the similarity of the regression coefficients and the material types (see Appendix S1, Section S1). Thus $m=31$ in Equation (1), with polyethylene terephthalate (PET) as the $32 \mathrm{nd}$ and reference material, as it is the material with most reported diffusion coefficients.

In previous studies, either the chemical's molecular weight (MW), molar volume (MV) or vapor pressure (VP) has been used as predictor of the diffusion coefficient in a given material. ${ }^{12,17-19}$ Begley et al. ${ }^{4}$ also suggested that the logarithm of the diffusion coefficient varies linearly with the inverse of the absolute temperature $(1 / T)$. Thus, the initial regression was performed to identify which of the above variables (MW, MV, VP, and $1 / T$ ) are best predictors of the diffusion coefficients of compounds encapsulated in the 32 material types, that is, to identify $X_{1}$ to $X_{n}$ in Equation (1). Details of the initial regression process are presented in Appendix S1, Section S2. Results of the initial regression model suggest that the log-molecular weight and the inverse of the absolute temperature are the most important predictors, and therefore the employed MLR model takes the following form:

$$
\log _{10} D=\alpha+\beta_{\log _{10} \mathrm{MW}} \cdot \log _{10} \mathrm{MW}+\beta_{1 / \mathrm{T}} \cdot \frac{1}{T}+b_{1} \cdot M_{1}+\ldots+b_{\mathrm{m}} \cdot \mathrm{M}_{\mathrm{m}}
$$

where $\mathrm{MW}$ is the chemical's molecular weight $(\mathrm{g} / \mathrm{mol})$ and $T$ is the absolute temperature (K).

The model performance of using log-molecular weight and molecular weight as predictors were very close when using the training dataset (1103 records, $m=31$ ), but the model using log-molecular weight as predictor was finally selected as it performs better for high-molecularweight chemicals (Section 3.3.3).

\subsection{3 | Temperature dependence}

Studies have shown that the activation energy of diffusion is a contributor to the temperature dependence of the diffusion coefficient and varies as function of both the material and the chemical properties. ${ }^{4,30,31}$ Thus, ideally a specific temperature correction coefficient should be used for each chemical-material combination. As data availability is not sufficient to determine chemical-specific temperature coefficients for each of the 32 materials, and as chemical properties seem to have limited influence on the activation energy, ${ }^{4,30}$ we followed the strategy of Begley et al., ${ }^{4}$ differentiating temperature coefficients for a limited number of material groups, applying one generic temperature coefficient for all chemicals within each material group. Begley et al. ${ }^{4}$ have introduced a variable $\tau$ to adjust the 
temperature coefficient for two groups of materials, where $\tau$ equals 0 or 1577 for nine different polymers, which corresponds to activation energy of $86.9 \mathrm{~kJ} / \mathrm{mol}$ for e.g. LDPE or $100 \mathrm{~kJ} / \mathrm{mol}$ for e.g. HDPE. To analyze the temperature dependency of the diffusion coefficients in our dataset, we first plotted $\log _{10} D$ against $1 / T$ for each of the 32 material types (Appendix S1, Section S3). The plots generally show as expected ${ }^{4}$ an inverse relationship in which $\log _{10} D$ is decreasing with increasing $1 / T$, different materials exhibiting different slopes. As variability in diffusion coefficient is higher between than within given studies, we first determined a temperature coefficient for each chemical-material type-study combination, and then calculated an average temperature coefficient for each material type by averaging all temperature coefficients belonging to the same material type. The analysis of the material-specific temperature coefficients showed that the material types can be grouped into three categories: (i) high-, (ii) medium-, and (iii) low-coefficient categories, with three corresponding values for the temperature coefficient adjustment factor $\tau$, which are given later in Section 3.1. Details are presented in Appendix S1, Section S3.3. The adjusted MLR model takes the following form accordingly:

$$
\log _{10} D=\alpha+\beta_{\log } M W \cdot \log _{10} M W+\frac{\beta_{1 / T}+\tau}{T}+b_{1} \cdot M_{1}+\ldots+b_{m} \cdot M_{m}
$$

\subsection{4 | Final regression}

To avoid multicollinearity problems in the MLR model and to avoid the influence of the material type "Limited-data material group" on the temperature coefficients, we fixed the temperature coefficients determined using Equation (3) and thus the final regression takes the following form:

$$
\log _{10} D-\frac{\beta_{1 / T}+\tau}{T}=\alpha+\beta_{\log } \mathrm{MW} \cdot \log _{10} \mathrm{MW}+b_{1} \cdot \mathrm{M}_{1}+\ldots+b_{\mathrm{m}} \cdot \mathrm{M}_{\mathrm{m}}
$$

where the dependent variable is $\log _{10} D-\left(\beta_{1 / T^{+}} \tau\right) / T$ instead of $\log _{10} D$, with the values of $\beta_{1 / T}$ and $\tau$ obtained from Equation (3) and presented later in Section 3.1. In this final regression, all 1103 records of measured $D$ data were utilized including the material type "Limited-data material group," leading to $m=31$ material types, plus one reference material type, PET, with $b_{\mathrm{PET}}=0$.

\section{3 | Model validation}

Validation of the final MLR model (Equation 4) was performed using the QSARINS software, version 2.2.1 (www.qsar.it) which is developed by Gramatica et al. ${ }^{32,33}$

\subsection{1 | Internal validation}

The MLR model's capacity to predict portions of the training dataset was evaluated in an internal validation process, using two techniques for internal validation in QSARINS. The first one is the leave-more-out (LMO) cross-validation technique, which iteratively and randomly exclude a certain percentage of the measured diffusion coefficient data, and then computes the regression coefficients with the remaining data and uses those coefficients to make predictions for the excluded ones. ${ }^{33}$ We used 1000 iterations and the percentage of the excluded elements was set as $20 \%$.

The second technique for internal validation is the Y-scrambling procedure, which demonstrates that the model is not the result of chance correlation. In this procedure, the experimental responses (in our study, the temperature-adjusted diffusion coefficients) are shuffled at random and used with the original predictors to establish an MLR model. If the original MLR model is internally valid, the performances of the scrambled models should be much worse than the original model. ${ }^{33}$ We used 1000 iterations for the Y-scrambling.

\subsection{2 | External validation}

We also evaluated the model ability to provide reliable predictions on new datasets in a so-called external validation process, using the following two approaches.

The first approach was to split the existing dataset (1103 records) into one training dataset and one prediction datasets. The training dataset was used to generate regression coefficients of the MLR model, and then, the MLR model was applied to the prediction set to examine the prediction performances of the model. Three kinds of splitting were performed using existing options in the QSARINS software (see Appendix S1, Section S5.1 for details) by random percentage ( $20 \%$ of the entire dataset randomly selected as the prediction set, $80 \%$ rest to the training set), by response and by structure (data first ordered by responses of the temperatureadjusted diffusion coefficient, or by the first axis of principal component analysis (PCA) of the descriptors, respectively). We introduced a fourth kind of splitting by studies, as variability across studies for a given material is in general larger than variability within a given study, yielding similar sample sizes of approximately 880 data for the training set and 220 data for the prediction set (Appendix S1, Table S3).

The second approach of external validation was to use the entire collected dataset (1103 records) as the training set and to use an entirely separate dataset as the prediction set. For the prediction set, two datasets were used. The first one is a database of diffusion coefficients from the United States Food and Drug Administration (FDA), which is a "database available upon request" for guidance for industry (http://www.fda.gov/Food/ucm081818.htm), and includes nonpeer-reviewed diffusion coefficient data reported by industry. This dataset includes 191 records of experimental diffusion coefficients of 46 chemicals in 22 materials which are mainly polymers used for FCM (Appendix S1, Section S5.1). The quality and reliability of these data are not characterized by FDA. The second prediction dataset is constructed from several studies conducted before $1982,{ }^{34-36}$ referenced in an EPA report. ${ }^{37}$ This dataset, designated as "Data by 1982 ," includes 281 records of measured diffusion coefficients of 92 chemicals in eight polymer materials, also including self-diffusion (Appendix S1, Section S5.1). Data for both prediction sets are provided in Appendix S2. 


\subsection{3 | Applicability domain}

The analysis and definition of the applicability domain (AD) of models is a fundamental issue that must be addressed in QSAR and QPPR studies. The study of $A D$ can provide information on the reliability of the model predictions, that is, if the chemicals are inside the $A D$, the predictions are interpolated and are more reliable; if the chemicals are outside the $A D$, the predictions are extrapolated and less reliable, because effects can occur outside the AD that do not exist within the AD. ${ }^{38}$ Three complementary methods were applied to define the AD of the diffusion coefficient QPPR: the range of model predictors, the leverage approach, and the PCA of the model predictors. ${ }^{39}$ More explanation of these methods is provided in Appendix S1, Section S6.1. In our analysis, chemicals are considered inside the AD if they are viewed inside $A D$ by all three methods, whereas chemicals are considered outside $A D$ if they are viewed outside $A D$ by all three methods, and finally chemicals that fall inside the AD for only one or two methods are considered as "borderline".

\section{3 | RESULTS AND DISCUSSION}

\section{1 | Temperature dependence of the diffusion coefficient}

The compiled dataset of 1103 records including 158 chemicals and 32 material types shows that the diffusion coefficient in solid materials decreases with decreasing temperature, as demonstrated by the highly significant negative regression coefficient for the variable $1 / T$, with $\beta_{1 / T}=-4440(K)$ with a standard error (SE) of $164(K)$ and $P<.001$ in Equation 2 (Appendix S1, Section S3.1). This is in agreement with previous studies. $^{4,30,31}$ This general tendency of decreasing diffusion with increasing $1 / T$ is well illustrated by the example of PET, the material with the most data available (Figure $1 \mathrm{~A}$ - see Appendix S1, Figure S1 for other materials). To further refine the coefficient for the temperature variable into specific materials groups, Figure $1 \mathrm{~B}$ illustrates well for methyl methacrylate (MMA) homopolymer the importance of first determining a temperature coefficient for each separate study and material-chemical combination (Section 2.2.3) and then averaging the temperature coefficients across studies. The molecular weightnormalized diffusion coefficients show a negative linear relationship with $1 / T$ within each of the three experimental studies of Figure $1 \mathrm{~B},{ }^{40-42}$ with similar regression coefficients of $-4530(K),-5704(K),-3415(K)$, averaging $-4550(\mathrm{~K})$ with an SE of $305(\mathrm{~K})$. However, as the absolute $\log _{10} \mathrm{MW}$-normalized diffusion coefficients reported by Hennebert et al. ${ }^{42}$ are much higher than those reported by the other two studies, doing one regression with all data from the three studies would result in a non-significant temperature coefficient ( $P$-value of .19), thus demonstrating the importance to first perform temperature regressions using data from the same study and for the same chemical.

Table 1 presents the average temperature coefficients and their standard errors for each of the 32 consolidated material types. Based on the values of the temperature coefficients (unit in K), the 32 material types can be grouped into three categories: (i) high-coefficient
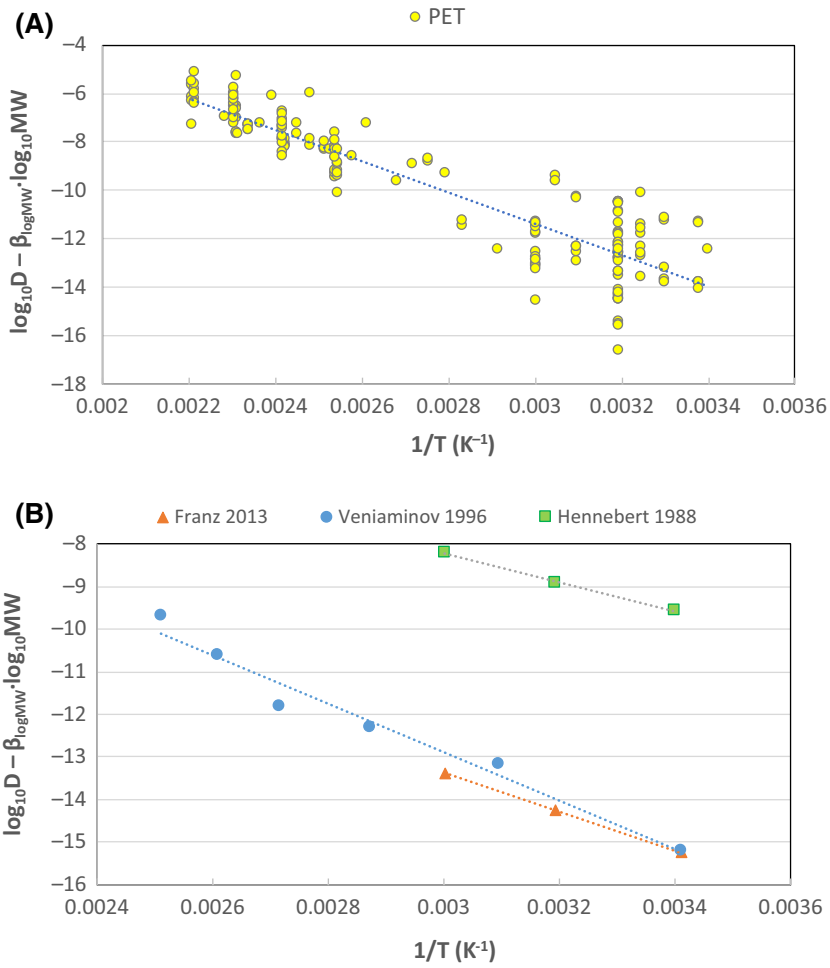

FIGURE 1 Relationship between the diffusion coefficient $D$ (corrected for $\log _{10} \mathrm{MW}$ ) and the inverse of temperature for (A) polyethylene terephthalate (PET), and (B) methyl methacrylate (MMA) homopolymer. The units of $D$ and molecular weight $(\mathrm{MW})$ are $\mathrm{m}^{2} / \mathrm{s}$ and $\mathrm{g} / \mathrm{mol}$, respectively

category with relatively high (absolute value) temperature coefficients $(<-5000)$, that is, materials in which diffusion coefficients are highly sensitive to the change in temperature, (ii) medium-coefficient category with temperature coefficients in between $\left(-5000<\left(\beta_{1 / T}+\tau\right)<-3000\right)$, and (iii) low-coefficient category with relatively low (absolute value) temperature coefficients (>-3000), that is, materials in which diffusion coefficients are least sensitive to the change in temperature. Details for the grouping of temperature coefficients can be found in Appendix S1, Section S3.3.

The temperature coefficients $\beta_{1 / T}$ and $\tau$ used in Equation (4) for each of the three temperature dependency material categories are obtained from the regression using the MLR model of Equation S3-2 (Appendix S1, Section S3.3), yielding values of $\beta_{1 / T}=-3486 \pm 299(K)$ and $\tau_{\text {high }}=-2391 \pm 356(K), \tau_{\text {medium }}=0(K)$ and $\tau_{\text {low }}=+1676 \pm 510(K)$. Thus, for the high-, medium-, and low-coefficient categories, the final temperature coefficients $\left(\beta_{1 / T+} \tau\right)$ are $-5877(K),-3486(K)$, and $-1810(K)$, corresponding to activation energy of $113,66.7$, and $34.7(\mathrm{~kJ} / \mathrm{mol})$, respectively. Begley et al. ${ }^{4}$ also aggregated nine types of polymer materials into two temperature categories, with activation energy of 100 and $86.9(\mathrm{~kJ} / \mathrm{mol})$, which have similar values with the high- and medium-coefficient categories in the present paper, to which these nine polymer materials are assigned. These results indicate that the categorization of the temperature coefficient in the present paper is consistent with previous studies, while extending the QPPR to a wider range of materials. 
TABLE 1 Temperature dependence of diffusion coefficient in the 32 consolidated material types (all numbers are in the unit of K)

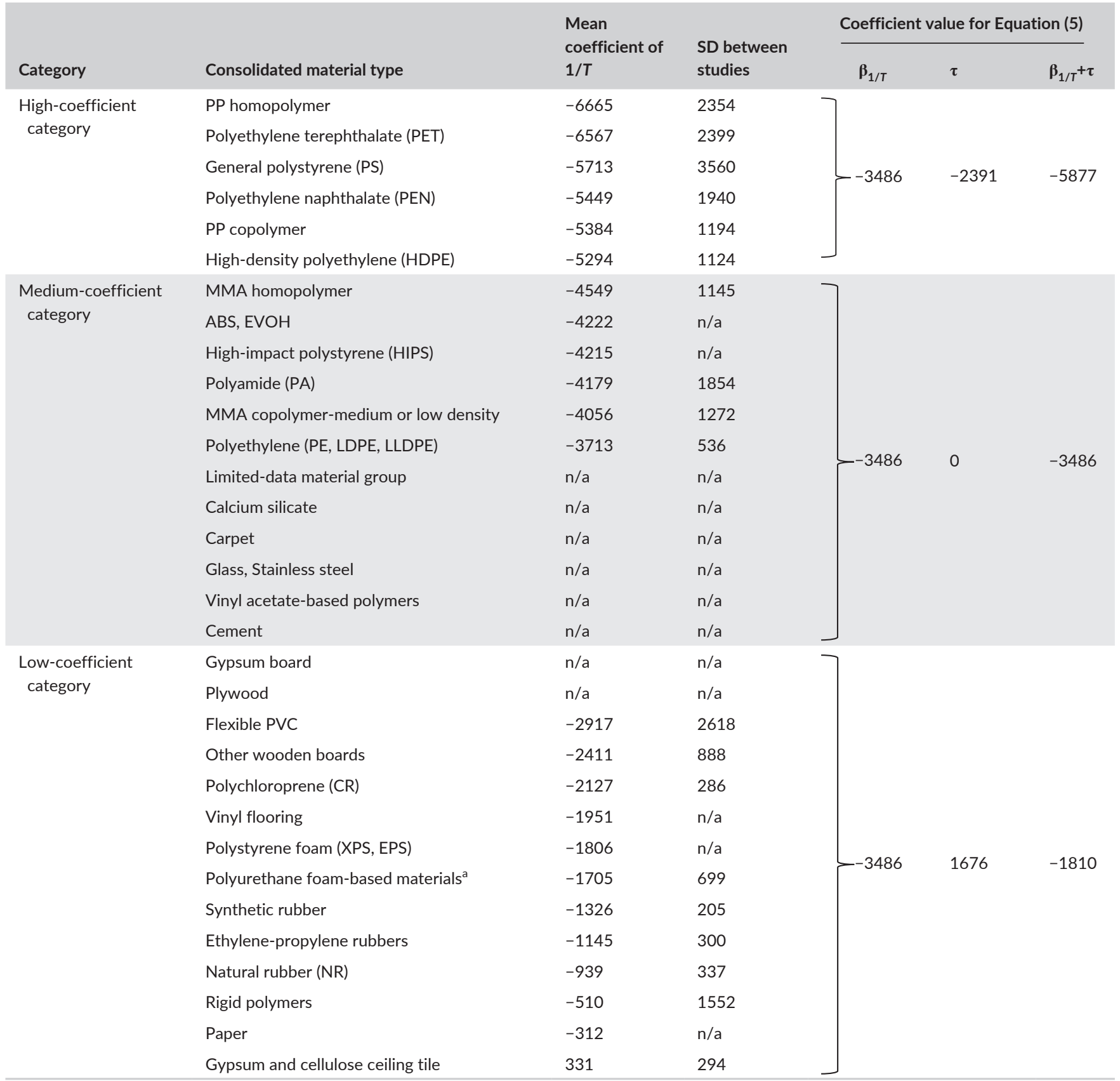

${ }^{a}$ This material type refers to low-density polyurethane foams with a density of $0.005-0.03 \mathrm{~g} / \mathrm{cm}^{3}$.

\section{2 | Final QPPR and model fitting}

Using the full dataset (1103 records) and Equation (4), the final MLR model for predicting the diffusion coefficient in solid materials is as follows:

$$
\begin{gathered}
\log _{10} D-\frac{\tau-3486}{T}=6.39-2.49 \cdot \log _{10} \mathrm{MW}+b \\
N=1103, R^{2}=.932, R_{\mathrm{adj}}^{2}=.930, \mathrm{SE}=1.17, \mathrm{RMSE}=1.15
\end{gathered}
$$

$$
\text { ANOVA: } F=457, d f=32, P<.0001
$$

where $D$ is the diffusion coefficient $\left(\mathrm{m}^{2} / \mathrm{s}\right), M W$ is molecular weight (g/ mol), $T$ is absolute temperature $(\mathrm{K}), b$ and $\tau(\mathrm{K})$ are the material-specific coefficients presented in Table 2 . This model is provided as an excel model in Appendix S3 to facilitate application. The standard errors for the intercept (6.39) and the coefficient of $\log _{10} \mathrm{MW}(-2.49)$ are 0.29 and 0.13 , respectively. An SE of 1.17 of the final model (Equation 5) indicates that the $95 \%$ confidence interval $(\mathrm{Cl})$ of the predicted response, $\log _{10} D-(\tau-3486) / T$, is the predicted value \pm 2.30 . The $95 \% \mathrm{Cl}$ of the $\log _{10} D$ cannot be directly calculated, but the average absolute difference between predicted and measured $\log _{10} D$ is 0.83 across the whole dataset (1103 records), and $95 \%$ of this absolute difference is below 2.54 . 
TAB LE 2 Material-specific coefficients for Equation (5)

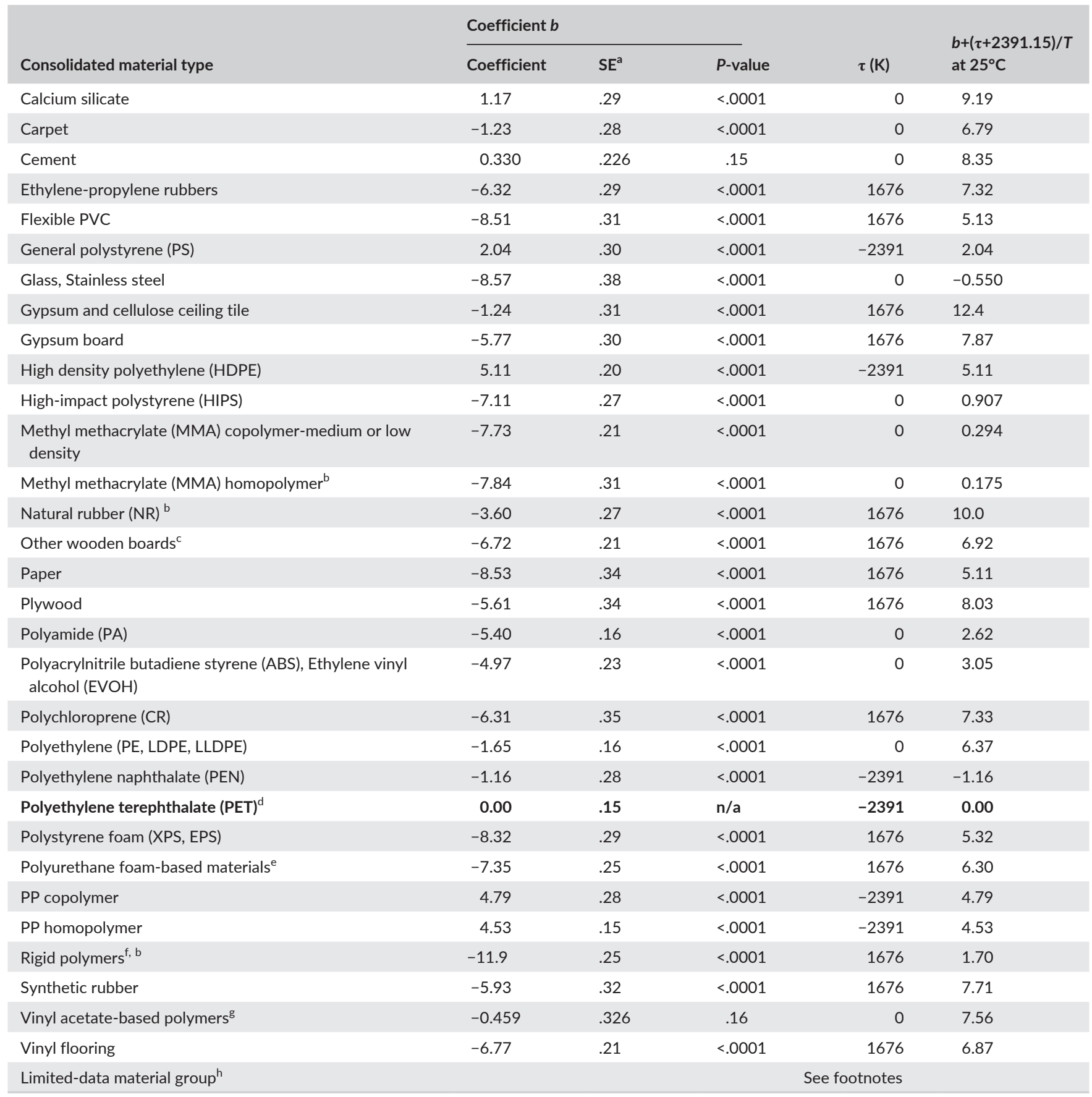

${ }^{\mathrm{a} S t a n d a r d ~ e r r o r . ~}$

${ }^{\mathrm{b} C}$ Coefficients should be taken with care due to large variations between studies.

'Includes Particleboard, Oriented strand board (OSB), Medium-density fiberboard (MDF), High-density board, and Wood chamber wall.

${ }^{\mathrm{d}}$ Reference material (in bold).

${ }^{\mathrm{e}}$ This material type refers to low-density polyurethane foams with a density of 0.005 to $0.03 \mathrm{~g} / \mathrm{cm}^{3}$.

fIncludes polyether ether ketone (PEEK), rigid PVC, polytetrafluoroethylene (PTFE), and polycarbonate.

IIncludes ethyl vinyl acetate (EVA), polyvinyl acetate (PVA), and polyvinyl acetate polyacrylic acid copolymer.

${ }^{h}$ The coefficient $b$ for this group is -2.26 with an SE of 0.18 , and the coefficient $\tau$ is 0 . "Limited-data material group" includes data from 20 different materials, so the accuracy of the coefficients is low and they are not recommended for use in predicting diffusion coefficients. This group includes alginate film, balance, decorative and overlay layers of wooden flooring, cellulose, epichlorhydrin-dimethylamine polymer (EDP), epoxy/acryic copolymer, latex, MMA/ Butyl methacrylic (BMA) copolymer-very low density, nanocomposite polyamide, paint, pectin film, pectin/alginate composite film, polydimethylsiloxane (PDMS) membrane, polyisoprene (PI) membrane, polyoctenamer $(\mathrm{PO})$ membrane, polyoxymethyene, polytrimethylene terephthalate (PTT), polyvinylidene chloride (PVDC), and silicone. 
This MLR model shows excellent fitting of the experimental data, with an adjusted $R$-square of 0.932 and a root mean square error (RMSE) of 1.15. The model fit is highly significant with an ANOVA $P$-value smaller than .0001. Figure 2A shows the scatter plot of experimental vs predicted responses, which aligns well with the 1:1 line. In this MLR model, the response (dependent variable) is the temperatureadjusted log diffusion coefficient, that is, $\log _{10} D-(\tau-3486) / T$, instead of $\log _{10} D$, in order to fix the temperature coefficients and to avoid multicollinearity problems, as mentioned in Section 2.2.4. The residual plot (Figure 2B) shows that the residuals are distributed evenly throughout the dataset, again indicating the good fit of the linear model for the data.

The key predictors other than temperature in the MLR model are the material type and the molecular weight of the diffusing chemical. The regression coefficient when considering log-molecular weight is equal to -2.49 , indicating that the diffusion coefficient decreases with increasing molecular weight. This implies that larger molecules diffuse more slowly compared to smaller molecules in solid materials, which is intuitive and consistent with findings from previous studies. $4,12,17,18$ However, although the molecular weight is a highly significant predictor $(P<.0001)$, it explains $<10 \%$ of the total variance of the diffusion coefficient (Appendix S1, Section S4).

The 31 dummy variables for the material types reflect the material dependency and account for most of the total variance of the diffusion

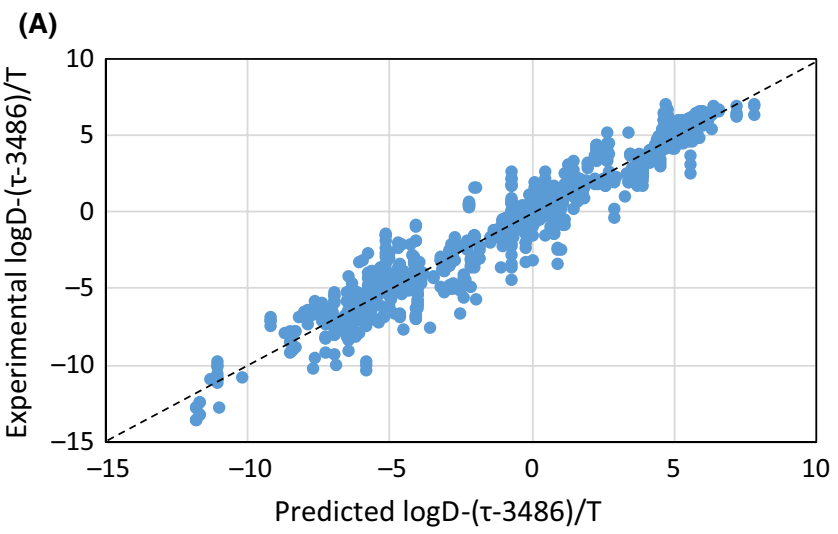

(B)

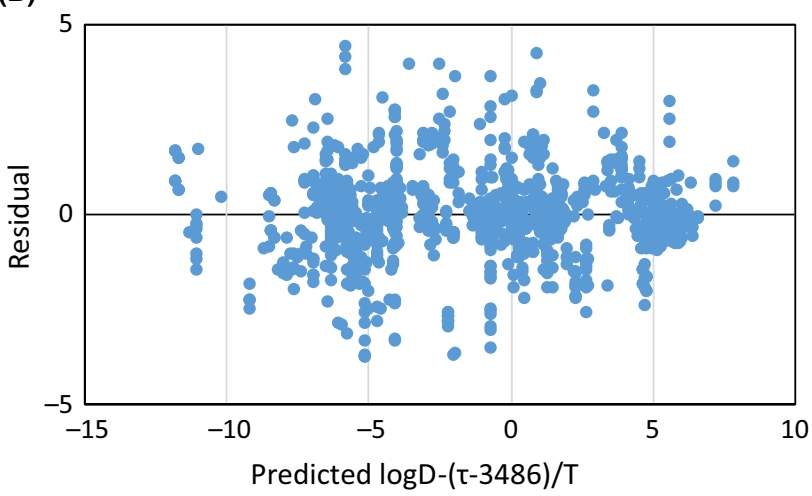

FIGURE 2 Values of $\log _{10} D-(\tau-3486) / T$ predicted by the final QPPR (Equation 5) vs (A) experimental values, and (B) residuals. The dotted line in $(A)$ indicates the 1:1 line. The units of $D$ and $T$ are $\mathrm{m}^{2} / \mathrm{s}$ and $\mathrm{K}$, respectively
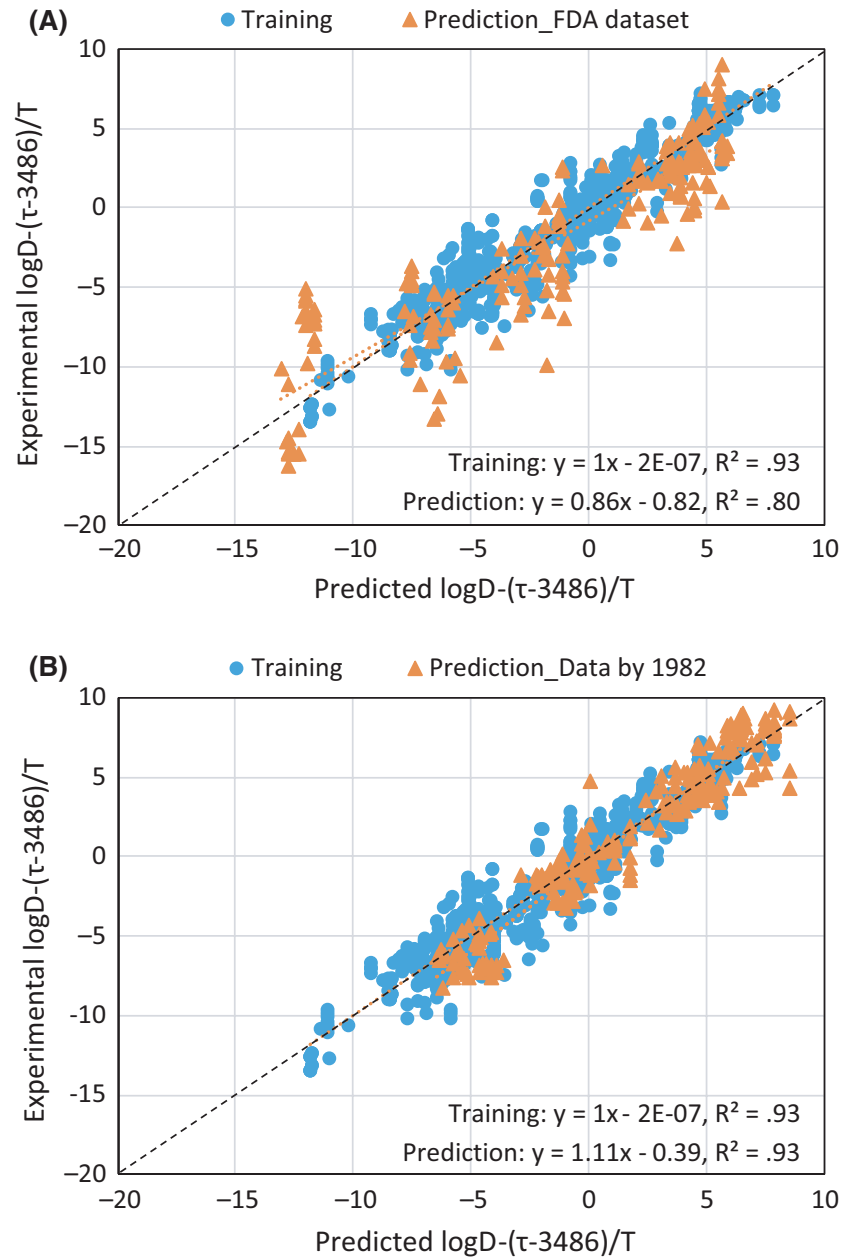

FIGURE 3 Values of $\log _{10} D-(\tau-3486) / T$ predicted by the final QPPR (Equation 5) vs experimental values when using (A) FDA dataset and (B) Data by 1982 as the prediction sets. The black dotted line indicates the 1:1 line. The units of $D$ and $T$ are $\mathrm{m}^{2} / \mathrm{s}$ and $K$, respectively

coefficient, indicating that the diffusion coefficient in solid materials is strongly dependent on the material type. As "PET" was used as the reference material in the regression, the value of its coefficient $b$ is zero (Table 2). For each of the other material types, the coefficient $b$, combined with the temperature coefficient $\tau$, that is, $b+(\tau+2391) / T$, determines the difference in log diffusion coefficient between that material type and PET, as PET has a temperature coefficient $\tau$ of -2391 (K) (Table 2, last column). Chemicals in material types with high values of $b+(\tau+2391) / T$ diffuse quicker than in material types with low values. Therefore, under room temperature $(T=298.15 \mathrm{~K})$, the values of $b+(\tau+2391) / T$ and the corresponding diffusion coefficients tend to be lower in dense, rigid materials such as glass, stainless steel, methyl methacrylate (MMA) polymers, polyethylene naphthalate (PEN), and rigid polymers including polyether ether ketone (PEEK), rigid PVC, polytetrafluoroethylene (PTFE), and polycarbonate (Table 2). In contrast, the values of $b+(\tau+2391) / T$ and the corresponding diffusion coefficients can be up to 13 orders of magnitude higher in flexible or porous materials, such as gypsum, wood, rubber, and polyurethane 
foam-based materials (Table 2). It should be noted that the composition and properties of a given material type may vary considerably depending on the intended use, as well as over time as material substitutions are made and production procedures differ. Thus, the material type coefficients in Table 2 actually represent an average composition and diffusion behavior for the specific material types.

The significance of the material type coefficient only indicates that the coefficient bs of these material types are significantly different from the reference material type, PET, but if another material type was selected as the reference material, the regression coefficients and statistical significance of all materials would change. Thus, the insignificance of the regression coefficients for material type variables does not indicate that those material types do not have a relevant influence on the diffusion coefficient. As a result, we keep all 31 material type dummy variables in the final regression to retain as much information as possible.

The MLR model given in Equation (5) contains material-specific variables, so it is only valid for the $\mathbf{3 2}$ material types presented in Table 2. For materials that do not belong to those 32 types, we built another generic QPPR to predict the diffusion coefficients, which is presented in Appendix S1, Section S4, which should be used with caution because of higher uncertainties.

\section{3 | Model validation results}

\subsection{1 | Internal validation}

For the $20 \%$ LMO cross-validation, the correlation coefficient, $Q^{2}{ }_{\text {LMO }}$ for the 1000 iterations ranges from 0.89 to 0.95 , with an average of 0.93 , and a root mean square error for cross-validation ( $\mathrm{RMSE}_{\mathrm{cv}}$ ) average of 1.19. Both the $Q^{2}{ }_{\text {LMO }}$ and $\mathrm{RMSE}_{\mathrm{CV}}$ are similar to the $R^{2}$ and RMSE computed using the full dataset, which is 0.93 and 1.15 , respectively. These results indicate that when fitted to a random $80 \%$ of the dataset the model is still able to predict the remaining $20 \%$ of the dataset, meaning that the model is internally stable.

For the Y-scrambling, the average $R_{\mathrm{Yscr}^{2}}^{2}$ and $Q^{2}{ }_{\mathrm{Yscr}}$ for the 1000 iterations are 0.029 and -0.033 , respectively, which are much smaller than the $R^{2}$ and $Q^{2}{ }_{L M O}$ of the original model. The RMSE for Y-scrambling, $R_{M S E}{ }_{Y_{s c r}}$, is 4.36 which is much higher than the RMSE and RMSE of the original model. These results demonstrate that no correlation exists between the scrambled responses and the predictors. Thus, chance correlation for the original model can be ruled out.

Overall, the internal validation demonstrates that the MLR model represented by Equation (5) is robust and stable, and is not a result of chance correlation.

\subsection{2 | External validation}

As described in Section 2.3.2, the first method of external validation was to split the full dataset (1103 records) into training set and prediction set, and four types of splitting were performed, including splitting by a random $20 \%$, by ordered response, by ordered structure, and by studies. Six criteria for external validation were computed and are presented in Table 3. The $R^{2}$ ext is the determination coefficient of the prediction set data using the model calculated using the training set data. The other five criteria, $Q^{2}{ }_{\mathrm{F} 1},{ }^{43} \mathrm{Q}_{\mathrm{F} 2}^{2},{ }^{44} \mathrm{Q}_{\mathrm{F} 3}{ }^{45}, \gamma_{\mathrm{m}}{ }^{46}$ and $\mathrm{CCC},{ }^{47}$ are external validation criteria proposed by different studies, which evaluate various aspects of the model's external prediction ability. These criteria are usually in accordance with each other but can sometimes give contradictory results, ${ }^{47}$ so they need to be evaluated together. Chirico and Gramatica ${ }^{48}$ have proposed threshold values for these different criteria, which are presented in Table 3. For the first three types of splitting (by random $20 \%$, by ordered response, and by ordered structure), the $R^{2}$ ext are higher than 0.9 , and all of the other five criteria pass the threshold values and are also higher than 0.9 , indicating good prediction ability of the model calculated using only the training set data.

\begin{tabular}{|c|c|c|c|c|c|c|}
\hline $\begin{array}{l}\text { External validation } \\
\text { criteria }\end{array}$ & $R^{2}{ }_{\text {ext }}$ & $Q^{2}{ }_{F 1}$ & $Q^{2}{ }_{F 2}$ & $Q^{2}{ }_{F 3}$ & $\overline{r_{\mathrm{m}}^{2}}$ & $\mathrm{CCC}$ \\
\hline Threshold & & $>.70$ & $>.70$ & $>.70$ & $>.65$ & $>.85$ \\
\hline $\begin{array}{l}\text { Splitting by random } \\
\text { percentage }\end{array}$ & .92 & .92 & .92 & .92 & .90 & .96 \\
\hline $\begin{array}{l}\text { Splitting by ordered } \\
\text { response }\end{array}$ & .94 & .94 & .94 & .95 & .93 & .97 \\
\hline $\begin{array}{l}\text { Splitting by ordered } \\
\text { structure }\end{array}$ & .94 & .94 & .94 & .94 & .91 & .97 \\
\hline Splitting by studies & .85 & .85 & .84 & .85 & .78 & .92 \\
\hline $\begin{array}{l}\text { FDA dataset as } \\
\text { prediction set }\end{array}$ & .80 & .77 & .77 & .60 & .71 & .89 \\
\hline $\begin{array}{l}\text { Data by } 1982 \text { as } \\
\text { prediction set }\end{array}$ & .93 & .93 & .92 & .90 & .85 & .95 \\
\hline
\end{tabular}

TABLE 3 External validation results

$R^{2}{ }_{\text {ext }}$, determination coefficient of the prediction set external data; $Q^{2}{ }_{F 1}$, correlation coefficient proposed by Shi et al.; $Q^{2}{ }_{F 2}$, correlation coefficient proposed by Schuurmann et al.; $Q^{2}{ }_{\mathrm{F} 3}$, correlation coefficient proposed by Consonni et al.; $r_{\mathrm{m}}^{2}$, determination coefficient proposed by Ojha et al.; CCC, concordance correlation coefficient proposed by Chirico and Gramatica. 
In these three types of splitting, the data were assigned to the training and prediction data sets either randomly or alternately (by ordered response or structure), so it is likely that a portion of the data from each study was assigned to the training set, while the remaining portion of the data was assigned to the prediction set. As a result, the prediction set is well within the AD defined by the training set (Appendix S1, Figures S2-S7), so it is expected that the model calculated using the training set can well predict the prediction set.

For the fourth type of splitting, splitting by studies, data from 30 studies were selected as the prediction set, while data from the remaining 48 studies constituted the training set. Thus, all data from one study and for one particular material will be either in the training or in the prediction set, so the validation using this splitting is close to a truly "external" validation. Most of the prediction set is inside the $A D$ defined by the training set except for two data points (Appendix S1, Figures S8-S9). As a result, the $R^{2}$ ext dropped to 0.85 , and the values of the other five validation criteria are apparently lower than those for the above three types of splitting, reflecting that variability is higher between than within studies. The five validation criteria nevertheless all pass the threshold values (Table 3), indicating that the model calculated using the training set has good prediction ability.

As a second method of external validation, the 1103 data points from the 68 studies were used as the training set, and additional data from an FDA database and from studies before 1982 were used as two separate prediction sets. As presented in Table 3, when using FDA dataset as the prediction set, the $R^{2}$ ext is reduced to 0.80 which is lower than the $R^{2}$ ext for the above four types of splitting. Four of the five validation criteria pass the threshold values, while $Q^{2}{ }_{\mathrm{F} 3}$ does not pass the threshold. In contrast, when using data by 1982 as the prediction set, the $R^{2}$ ext is 0.93 , which is very close to the $R^{2}$ of the training dataset (Section 3.2). The absolute difference between predicted and measured $\log _{10} \mathrm{D}$ averages 2.20 (95th percentile of 5.53) for the FDA dataset, and averages 1.08 (95th percentile of 2.68) for the data by 1982 . Figure 3 presents the comparison between model predicted and experimental responses for these two prediction sets. Data from both prediction sets are generally distributed close to the 1:1 line, but the FDA data are more dispersed compared to the training set data, while the data by 1982 are almost as compact as the training set data. The FDA data lack documentation of experimental details, so their quality may not be as good as the data reported in peer-reviewed literature. Also, when the FDA polymer types were linked to our consolidated material types, mismatches may have occurred due to lack of description of the polymers in the FDA dataset, which may lead to inaccuracies in model predictions. Overall, however, our QPPR performs reasonably well on these two fully external datasets, demonstrating its good predictive ability.

\subsection{3 | Applicability domain}

We performed the analysis of the model's AD using the three approaches explained in Section 2.3.3. The model being evaluated is the final MLR model presented in Equation (5), which was calculated using the training set of 1103 data points collected from 68 studies obtained from the peer-reviewed literature. For the analysis of $A D$, we focus on the two external prediction datasets: the FDA dataset (189 data points) and the data by 1982 (239 data points). Detailed results of the AD analysis are presented in Appendix S1, Section S6.1.

Combining the three methods, none of the data points in both prediction sets fell out of the AD. For the FDA dataset, the majority of the data points were inside the $A D$, while 15 data points were on borderline of AD. Similarly, only 35 data points from the data by 1982 were on borderline of AD. Thus, it is valid to use the present QPPR to make reliable estimates of diffusion coefficients for all data points in the two prediction sets. The physiochemical property space covered by the QPPR is mainly determined by the chemical's molecular weight, which ranges from 30 to $1178 \mathrm{~g} / \mathrm{mol}$. The vapor pressure at $25^{\circ} \mathrm{C}$ may also be a relevant property, which ranges from $9.8 \cdot 10^{-29}$ to $5.2 \cdot 10^{5} \mathrm{~Pa}$. The range of $\log _{10} D$ covered by the QPPR is from -22.1 to -5.2 where $D$ is measured in $\mathrm{m}^{2} / \mathrm{s}$.

As mentioned in Section 2.2.2, the model performances of using log-molecular weight and molecular weight as predictors were very close to each other when using the training dataset. However, residual analysis and external validation showed that $\log _{10} \mathrm{MW}$ is a more stable predictor than MW when handling high-molecular-weight chemicals, which becomes prominent for the FDA dataset, which includes certain chemicals with molecular weight higher than $1500 \mathrm{~g} / \mathrm{mol}$. While none of the data points in the FDA dataset fell out of the AD using the $\log _{10} \mathrm{MW}$ model, 11 data points would be outside AD using the MW model. Details are presented in Appendix S1, Section S6.2. Thus, $\log _{10} \mathrm{MW}$ instead of $\mathrm{MW}$ was selected as a predictor in the final QPPR (Equation 5).

Schwope et al. ${ }^{37}$ suggested that the linear relationship between $\log _{10} D$ and $\log _{10}$ MW may only be valid for a certain range of molecular weight, and there may be a saturation of diffusion coefficients for small molecular weights, that is, for a given material and a given temperature, the diffusion coefficient does not continue to increase for chemicals with molecular weight lower than a certain value, which is likely determined by the material type. To further examine the effect of molecular weight on model applicability, we analyzed the model residuals vs the log of molecular weight for the training dataset and the two prediction sets (Figure 4). For the three datasets, the residuals are distributed evenly on both sides of zero in the MW range of the training dataset of 30 to $1178 \mathrm{~g} / \mathrm{mol}\left(\log _{10} \mathrm{MW}\right.$ of 1.48-3.07). For methane $(\mathrm{MW}=16 \mathrm{~g} / \mathrm{mol})$, most of the predictions overestimate diffusivity, suggesting that diffusivity may indeed not further decrease below $\mathrm{MW}$ of $30 \mathrm{~g} / \mathrm{mol}$. As methane was the only chemical with data available for $\mathrm{MW}$ lower than $30 \mathrm{~g} / \mathrm{mol}$, data for additional chemicals and materials are therefore needed to further test this hypothesis of saturation at low MW. Similarly, additional data are needed to provide more accurate estimates for chemicals with very high molecular weights.

Overall, the performance of the final model (Equation 5) in this external validation indicates that it has the ability to provide reliable predictions, as long as the considered chemicals are within the model's $A D$. With the log-molecular weight as a predictor, our model is able to make reliable extrapolations on chemicals with molecular weights up to about $2500 \mathrm{~g} / \mathrm{mol}$, but caution still needs to be taken when applying 


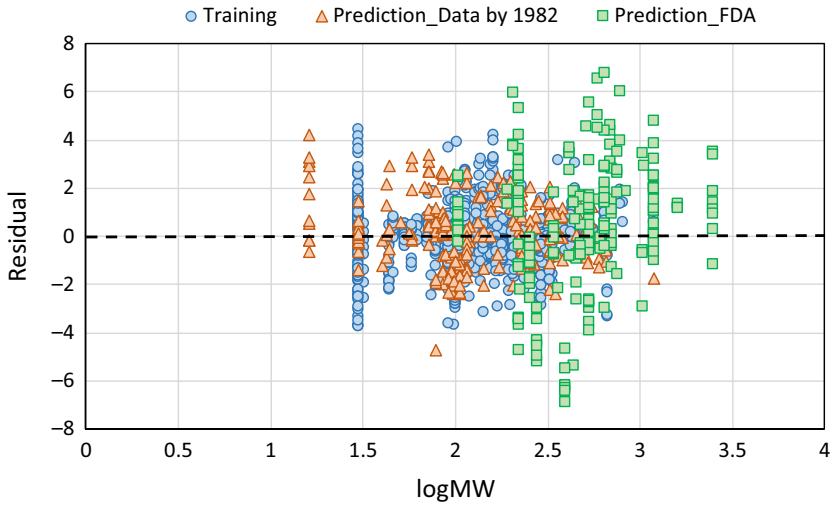

FIGURE 4 Residual between the present QPPR and observed data as a function of $\log _{10} \mathrm{MW}$ for the training dataset, the FDA dataset, and the data by 1982 set. The unit of $\mathrm{MW}$ is $\mathrm{g} / \mathrm{mol}$

the model on extremely high-molecular-weight chemicals. Ideally, the model should be applied to predict diffusion coefficients for chemicals with molecular weights lower than $1178 \mathrm{~g} / \mathrm{mol}$ which is the maximum within the training dataset. Caution also needs to be taken when applying the model on very-low-molecular-weight chemicals due to the possible saturation effect. Both the FDA dataset and the data by 1982 were used for the external validation but not combined with the original training dataset to calculate a more comprehensive MLR model, because these data are somewhat outdated; the FDA data are not published in literature, so there is a lack of experimental details, making these undocumented data less reliable than the data collected from peer-reviewed literature.

\subsection{Limitations and future work}

While the extension to 32 different consolidated material types is a major progress, the present model is still not fully comprehensive. First, the model may not be valid for very high or very low molecular weight (MW) chemicals. It may not be valid for ionizing organic chemicals either, as ionizing chemicals such as acids, alcohols/phenols, and amines are not well represented in the training dataset, as they only account for $<10 \%$ of the data points, and the model does not consider chemical ionization or interaction within a material, which may make the chemical's diffusivity lower than that predicted by the model. Second, the present model is not applicable for materials types other than the 32 types in the training set, for example, for material such as resin and textiles, due to the lack of experimental data. Although a more general MLR model (Appendix S1, Section S4) was developed which does not require material type as the predictor, it gives much less accurate predictions of the diffusion coefficient. Third, the present model does not consider any interaction between $\mathrm{MW}$ and material type, that is, it assumes the effect of MW is the same across different materials. Although model validations show that this assumption may be reasonable for the existing data, ideally it needs to be further verified using data spanning the whole $\mathrm{MW}$ range $(30-1178 \mathrm{~g} / \mathrm{mol})$ for each material. Therefore, more experimental diffusion coefficient data need to be obtained, or more advanced experimental methods to measure diffusion coefficients need to be developed, for other material types and chemical sizes and classes to make the model more comprehensive.

There are also large variations in the experimental diffusion coefficients between some of different studies for three material types, namely "MMA homopolymer," "Natural rubber," and "Rigid polymers," even after correcting for molecular weight and temperature, as shown in Figure 1 and Appendix S1, Figure S1. This means that the regression coefficients $b$ and $\tau$ for these material types should be taken with care. The variations could be due to three causes. First, experimental variation, for example, Franz et al. ${ }^{40}$ used desorption experiments to measure the diffusion coefficients in MMA homopolymer, while Hennebert et al. ${ }^{42}$ used sorption experiments. Second, the swelling of polymers during liquid sorption experiments, which generally occurs for cross-linked polymers in low-molecular weight solvents, ${ }^{49}$ may not always be accounted for, and can lower the diffusion coefficients by orders of magnitude. ${ }^{10}$ Third, the properties of the same material can vary between studies depending on how it was made and which additives were used. This may also be the case for some other materials such as vinyl flooring, carpet, and synthetic rubber for which the material type coefficients in Equation (5) can only represent some sort of average composition and diffusion behavior for the specific materials. Ideally, quantitative, continuous properties of the solid materials, such as density, porosity, and crystalline state of the material as well as other descriptors of the material's composition and molecular structure, instead of qualitative material types could be measured and entered into the model as predictors, so that the model can be more accurate and can be extrapolated to various material types outside the training dataset.

\section{4 | CONCLUSIONS}

A MLR model has been developed to predict the internal diffusion coefficients of organic compounds in various solid materials (excel model provided in Appendix S3). Experimental diffusion coefficient data collected from 68 studies of the peer-reviewed literature were used as the training set for the regression. The model uses two continuous variables, molecular weight and inversed absolute temperature, and one categorical variable, material type, as predictors. The model has been internally validated to be robust, stable and not a result of chance correlation. External validation using two prediction sets demonstrates that the model predictions are most reliable within the model's AD, namely molecular weight between 30 and $1178 \mathrm{~g} / \mathrm{mol}$, temperature between 4 and $180^{\circ} \mathrm{C}$, and material type belonging to the 32 consolidated types.

The main advantage of the present model is that it is applicable for chemicals with a wide range of molecular weights (but only up to about $16-2500 \mathrm{~g} / \mathrm{mol}$, with special treatment for molecular weight lower than $30 \mathrm{~g} / \mathrm{mol}$ ) in various materials. This is advantageous compared to the correlation methods developed in previous studies often specific for certain chemical classes or materials. The present model is able to provide reliable estimates of diffusion coefficients for a large 
number of chemical-material combinations, making it suitable for high-throughput assessments of the releases and human exposures to chemicals encapsulated in solid materials, particularly building materials and FCMs. To make the model comprehensive, more experimental diffusion coefficient data need to be obtained for other material types, or quantitative and continuous parametrization of various solid materials needs to be further developed.

\section{ACKNOWLEDGEMENTS}

The authors thank Prof. Ester Papa, Dr Allessandro Sangion, and Prof. Paola Gramatica from the University of Insubria, Italy, for advice on MLR modeling and validation, as well as support for the QSARINS software. Funding for this research was provided by US EPA contract EP-16-C-000070 and by the Long Range Research Initiative of the American Chemistry Council. P. Fantke was supported by the Marie Curie project Quan-Tox (GA No. 631910) funded by the European Commission under the Seventh Framework Programme.

\section{REFERENCES}

1. Little JC, Weschler CJ, Nazaroff WW, et al. Rapid methods to estimate potential exposure to semivolatile organic compounds in the indoor environment. Environ Sci Technol. 2012;46:11171-11178.

2. $\mathrm{Xu} Y$, Cohen Hubal EA, Clausen PA, et al. Predicting residential exposure to phthalate plasticizer emitted from vinyl flooring: a mechanistic analysis. Environ Sci Technol. 2009;43:2374-2380.

3. Guo Z. Review of indoor emission source models. Part 1. Overview. Environ Pollut. 2002a;120:533-549.

4. Begley T, Castle L, Feigenbaum A, et al. Evaluation of migration models that might be used in support of regulations for food-contact plastics. Food Addit Contam. 2005;22:73-90.

5. Xie M, Wu Y, Little JC, et al. Phthalates and alternative plasticizers and potential for contact exposure from children's backpacks and toys. J Expo Sci Environ Epidemiol. 2016;26:119-124.

6. Liu Z, Ye W, Little JC. Predicting emissions of volatile and semivolatile organic compounds from building materials: a review. Build Environ. 2013;64:7-25.

7. Haghighat $\mathrm{F}$, Huang $\mathrm{H}$, Lee $\mathrm{C}$-S. Modeling approaches for indoor air VOC emissions from dry building materials-a review. ASHRAE Trans. 2005;111:635-645.

8. Berens A, Hopfenberg $\mathrm{H}$. Diffusion of organic vapors at low concentrations in glassy PVC, polystyrene, and PMMA. J Membr Sci. 1982;10:283-303.

9. Hickey AS, Peppas NA. Solute diffusion in poly (vinyl alcohol)/poly (acrylic acid) composite membranes prepared by freezing/thawing techniques. Polymer. 1997;38:5931-5936.

10. John J, Kunchandy S, Kumar A, et al. Transport of methyl methacrylate monomer through natural rubber. J Mater Sci. 2010;45:409-417.

11. Luo R, Niu J. Determining diffusion and partition coefficients of VOCs in cement using one FLEC. Build Environ. 2006;41:1148-1160.

12. Bodalal A, Zhang J, Plett E, et al. Correlations between the internal diffusion and equilibrium partition coefficients of volatile organic compounds (VOCs) in building materials and the VOC properties. ASHRAE Trans. 2001;107:789.

13. Bodalal A, Zhang J, Plett E. A method for measuring internal diffusion and equilibrium partition coefficients of volatile organic compounds for building materials. Build Environ. 2000;35:101-110.

14. Little JC, Hodgson AT, Gadgil AJ. Modeling emissions of volatile organic compounds from new carpets. Atmos Environ. 1994;28:227-234.
15. Dole P, Feigenbaum AE, Cruz CDL, et al. Typical diffusion behaviour in packaging polymers-application to functional barriers. Food Addit Contam. 2006;23:202-211

16. Reynier A, Dole P, Humbel S, et al. Diffusion coefficients of additives in polymers. I. Correlation with geometric parameters. J Appl Polym Sci 2001;82:2422-2433

17. Guo Z. Review of indoor emission source models. Part 2. Parameter estimation. Environ Pollut. 2002b;120:551-564.

18. Cox SS, Zhao D, Little JC. Measuring partition and diffusion coefficients for volatile organic compounds in vinyl flooring. Atmos Environ. 2001;35:3823-3830.

19. Zhao D, Cox S, Little J. Source/sink characterization of diffusion controlled building materials. In: Proceedings of the 8th International Conference on Indoor Air Quality and Climate-Indoor Air. 1999.

20. Jolliet O, Ernstoff AS, Csiszar SA, et al. Defining product intake fraction to quantify and compare exposure to consumer products. Environ Sci Technol. 2015;49:8924-8931.

21. Shin H-M, Ernstoff A, Arnot JA, et al. Risk-based high-throughput chemical screening and prioritization using exposure models and in vitro bioactivity assays. Environ Sci Technol. 2015;49:6760-6771.

22. Shin $\mathrm{H}-\mathrm{M}$, McKone TE, Bennett $\mathrm{DH}$. Intake fraction for the indoor environment: a tool for prioritizing indoor chemical sources. Environ Sci Technol. 2012;46:10063-10072.

23. Ernstoff AS, Fantke P, Csiszar SA, et al. Multi-pathway exposure modelling of chemicals in cosmetics with application to shampoo. Environ Int. 2016;92-93:87-96.

24. Csiszar SA, Ernstoff AS, Fantke P, et al. Stochastic modeling of nearfield exposure to parabens in personal care products. J Expo Sci Environ Epidemiol. 2017;27:152-159.

25. Egeghy PP, Sheldon LS, Isaacs KK, et al. Computational exposure science: an emerging discipline to support 21st-century risk assessment. Environ Health Perspect. 2016;124:697.

26. Deng Q, Yang X, Zhang J. Study on a new correlation between diffusion coefficient and temperature in porous building materials. Atmos Environ. 2009;43:2080-2083.

27. Xu J, Zhang JS. An experimental study of relative humidity effect on VOCs' effective diffusion coefficient and partition coefficient in a porous medium. Build Environ. 2011;46:1785-1796.

28. Xu J, Zhang JS, Liu X, et al. Determination of partition and diffusion coefficients of formaldehyde in selected building materials and impact of relative humidity. J Air Waste Manag Assoc. 2012;62:671-679.

29. Park J-S, Little JC, Kim S-D, et al. The determination of diffusion and partition coefficients of PUF. J Korean Soc Atmos Environ. 2010;26:77-84

30. Welle F, Franz R. Diffusion coefficients and activation energies of diffusion of low molecular weight migrants in Poly(ethylene terephthalate) bottles. Polym Test. 2012;31:93-101.

31. Ewender J, Welle F. Determination of the activation energies of diffusion of organic molecules in poly (ethylene terephthalate). J Appl Polym Sci. 2013;128:3885-3892.

32. Gramatica P, Cassani S, Chirico N. QSARINS-chem: insubria datasets and new QSAR/QSPR models for environmental pollutants in QSARINS. J Comp Chem. 2014;35:1036-1044.

33. Gramatica P, Chirico N, Papa E, et al. QSARINS: a new software for the development, analysis, and validation of QSAR MLR models. J Comp Chem. 2013;34:2121-2132.

34. Flynn JH. A collection of kinetic data for the diffusion of organic compounds in polyolefins. Polymer. 1982;23:1325-1344.

35. Park G. The diffusion of some organic substances in polystyrene. Trans Faraday Soc. 1951;47:1007-1013.

36. Park G. The diffusion of some halo-methanes in polystyrene. Trans Faraday Soc. 1950;46:684-697.

37. Schwope A, Goydan R, Reid R. Methods for Assessing Exposure to Chemical Substances Volume 11: Methodology for Estimating 
the Migration of Additives and Impurities from Polymeric Materials. Washington, D.C.: U.S.EPA; 1990.

38. Gramatica P. Principles of QSAR models validation: internal and external. QSAR Comb Sci. 2007;26:694-701.

39. Cassani S, Gramatica P. Identification of potential PBT behavior of personal care products by structural approaches. Sustain Chem Pharm. 2015;1:19-27.

40. Franz R, Brandsch R. Migration of acrylic monomers from methacrylate polymers-establishing parameters for migration modelling. Packag Technol Sci. 2013;26:435-451.

41. Veniaminov A, Sedunov YN. Diffusion of phenanthrenequinone in poly (methyl methacrylate): holographic measurements. Polym Sci Ser A. 1996;38:59-63.

42. Hennebert P. Solubility and diffusion coefficients of gaseous formaldehyde in polymers. Biomaterials. 1988;9:162-167.

43. Shi LM, Fang $\mathrm{H}$, Tong $\mathrm{W}$, et al. QSAR models using a large diverse set of estrogens. J Chem Inf Comput Sci. 2001;41:186-195.

44. Schüürmann G, Ebert R-U, Chen J, et al. External validation and prediction employing the predictive squared correlation coefficient Test set activity mean vs training set activity mean. J Chem Inf Model. 2008;48:2140-2145.

45. Consonni V, Ballabio D, Todeschini R. Comments on the definition of the $\mathrm{Q}^{2}$ parameter for QSAR validation. J Chem Inf Model. 2009;49:1669-1678.

46. Ojha PK, Mitra I, Das RN, et al. Further exploring $r_{m}{ }^{2}$ metrics for validation of QSPR models. Chemometr Intell Lab Syst. 2011;107:194-205.
47. Chirico N, Gramatica P. Real external predictivity of QSAR models: how to evaluate it? Comparison of different validation criteria and proposal of using the concordance correlation coefficient. J Chem Inf Model. 2011;51:2320-2335.

48. Chirico N, Gramatica P. Real external predictivity of QSAR models. Part 2. New intercomparable thresholds for different validation criteria and the need for scatter plot inspection. J Chem Inf Model. 2012;52:2044-2058.

49. Nandi S, Winter HH. Swelling behavior of partially cross-linked polymers: a ternary system. Macromolecules. 2005;38:4447-4455.

\section{SUPPORTING INFORMATION}

Additional Supporting Information may be found online in the supporting information tab for this article.

How to cite this article: Huang L, Fantke P, Ernstoff A, Jolliet $O$. A quantitative property-property relationship for the internal diffusion coefficients of organic compounds in solid materials. Indoor Air. 2017;27:1128-1140.

https://doi.org/10.1111/ina.12395 